\title{
New dextran coated activated carbons for medical use
}

\author{
C. A. Howell, S. R. Sandeman, Y. Zheng, S. V. Mikhalovsky, V. G. Nikolaev, L. A. Sakhno, E. A. Snezhkova
}

- School of Engineering

\begin{abstract}
Activated carbon (AC) can be used for blood purification (hemoperfusion) to treat a range of conditions by direct perfusion of blood through an adsorbent column. The clinical effects of hemoperfusion relate to the AC adsorptive capacity, and any inflammatory response generated by direct blood contact. The use of a biocompatible coating minimizes side-effects but at the same time significantly reduces AC adsorption capacity in particular towards substances with large molecular mass. We investigated the adsorption capacity for biological molecules in a wide range of molecular masses and biocompatibility of a dextran (Dx) coated mesoporous AC (HSGD) produced from a polymer precursor. The hemocompatibility of the Dxcoated beads was investigated according to the ISO guidelines. Coating the HSGD with a Dx coating (5-30\% weight of $\mathrm{AC}$ ) decreased the specific surface area and pore volume of the materials and reduced adsorption capacity for methylene blue, vitamin $B_{12}$, bilirubin and albumin, while minimizing fines production. Even the partially coated materials did not activate the inflammatory response and thus could be considered hemocompatible, with the Dx coating reducing fibrinogen adsorption and complement generation, suggesting the material warrants continued development for hemoperfusion applications.
\end{abstract}

Original language English

Pages (from-to) $\quad 134-146$

Number of pages 13

Journal $\quad \underline{\text { Carbon }}$

Volume 97

State $\quad$ Published - Feb 12016

Howell, C. A., Sandeman, S. R., Zheng, Y., Mikhalovsky, S. V., Nikolaev, V. G., Sakhno, L. A., \& Snezhkova, E. A. (2016). New dextran coated activated carbons for medical use. Carbon, 97, 134-146. DOI: 10.1016/j.carbon.2015.09.042 